\title{
VARIATIONS ON THE THEME OF HILBERT'S THEOREM 90
}

\author{
A. DUBICKAS \\ Department of Mathematics and Informatics, Vilnius University, Naugarduko 24, Vilnius 2006, Lithuania \\ e-mail: arturas.dubickas@maf.vu.lt \\ and C. J. SMYTH \\ School of Mathematics, University of Edinburgh, James Clerk Maxwell Building, \\ King's Buildings, Mayfield Road, Edinburgh EH9 3JZ, UK \\ e-mail: chris@maths.ed.ac.uk
}

(Received 14 December, 2000; accepted 2 February, 2001)

\begin{abstract}
Given a number field $K$, we find two simple separate necessary and sufficient conditions on a given algebraic number for it to be expressible as a quotient (respectively as a difference) of two algebraic numbers conjugate over $K$.
\end{abstract}

2000 Mathematics Subject Classification. 11R06, 12F05.

1. Introduction. Let $K$ be a fixed number field. Which algebraic numbers $\beta$ can be written as a quotient $\alpha / \alpha^{\prime}$ of algebraic numbers $\alpha$ and $\alpha^{\prime}$ that are conjugate over $K$ ? Since some non-zero rational power of $\operatorname{Norm}_{K}\left(\alpha / \alpha^{\prime}\right)$ is $\operatorname{Norm}_{K}(\alpha) /$ $\operatorname{Norm}_{K}\left(\alpha^{\prime}\right)=1$, it is clear that $\operatorname{Norm}_{K}(\beta)$ must be a root of unity in order that $\beta=\alpha / \alpha^{\prime}$. But it turns out that this norm condition is not sufficient for $\beta=\alpha / \alpha^{\prime}$, as we show in Theorem 1.1 below, where a necessary and sufficient condition on $\beta$ is given. Here as usual $\operatorname{Norm}_{K}(\gamma)$, and $\operatorname{Trace}_{K}(\gamma)$ below, denote respectively $\operatorname{Norm}_{K(\gamma) / K}(\gamma)$ and $\operatorname{Trace}_{K(\gamma) / K}(\gamma)$, the product and sum of the conjugates of $\gamma$ over $K$.

Similarly, if an algebraic number $\beta$ is a difference $\alpha-\alpha^{\prime}$, where $\alpha$ and $\alpha^{\prime}$ are conjugate over $K$, then since $\operatorname{Trace}_{K}\left(\alpha-\alpha^{\prime}\right)=0$, $\operatorname{Trace}_{K}(\beta)$ must also be zero. Again, however, this condition is not sufficient for $\beta$ to be such a difference. A necessary and sufficient condition on $\beta$ for this to occur is given in Theorem 2.1.

In order to state our main results, we need some notation. For an algebraic number $\gamma$ we denote by $N_{\gamma}$ the normal closure of the field $K(\gamma)$ over $K$, with Galois group $G_{\gamma}=\operatorname{Gal}\left(N_{\gamma} / K\right)$. For $\sigma \in G_{\gamma}$ and $\delta \in N_{\gamma}$ we denote by $O(\sigma, \delta)$ the orbit of $\delta$ under the action of the cyclic group $\langle\sigma\rangle$ generated by $\sigma$, and put

$$
P(\sigma, \delta)=\prod_{\delta^{\prime} \in O(\sigma, \delta)} \delta^{\prime}, \quad S(\sigma, \delta)=\sum_{\delta^{\prime} \in O(\sigma, \delta)} \delta^{\prime} .
$$

We can now state our first result.

Theorem 1.1. Let $K$ be a given number field. A non-zero algebraic number $\beta$ can be written as a quotient $\alpha / \alpha^{\prime}$ of algebraic numbers $\alpha$ and $\alpha^{\prime}$ conjugate over $K$ if and only if there is a $\sigma \in G_{\beta}$ such that $P(\sigma, \beta)$ is a root of unity. 
Moreover, if $\beta$ is a quotient $\alpha / \alpha^{\prime}$ of algebraic numbers conjugate over $K$, with say $P(\sigma, \beta)$ an $\ell$ th root of unity, $n=|\langle\sigma\rangle|, m=|O(\sigma, \beta)|$ and $k=\ell / \operatorname{gcd}(\ell, n / m)$, then $\alpha$ and $\alpha^{\prime}$ can be chosen such that $\alpha^{k}$ lies in $N_{\beta}$ (and hence so also does $\alpha^{\prime k}$ ).

As consequences of this result, we have the following corollaries.

COROLlary 1.2. (Hilbert's Theorem 90 [4],[5]) Suppose that $L / K$ is a cyclic Galois extension and that $\beta \in L$. Then $\beta$ is a quotient $\alpha / \alpha^{\prime}$, for some $\alpha, \alpha^{\prime} \in L$, conjugate over $K$, if and only if $\operatorname{Norm}_{L / K}(\beta)=1$.

For if $\operatorname{Gal}(L / K)=\langle\sigma\rangle$ with $n=|\langle\sigma\rangle|=[L: K]$ and $\beta=\alpha / \tau \alpha$, for some $\alpha \in L$, $\tau \in\langle\sigma\rangle$ then $\operatorname{Norm}_{L / K}(\beta)=\prod_{i=1}^{n} \tau^{i-1} \alpha / \prod_{i=1}^{n} \tau^{i} \alpha=1$. Conversely, if $\operatorname{Norm}_{L / K}(\beta)=1$, then, for $\ell=n / m, \operatorname{Norm}_{K}(\beta)=P(\sigma, \beta)$ is an $\ell$ th root of unity, so that we can apply Theorem 1.1 with $k=1$.

Corollary 1.3. If $|O(\sigma, \beta)|=\operatorname{deg} \beta$, for some $\sigma \in N_{\beta}$, and $\operatorname{Norm}_{K}(\beta)$ is a root of unity, then $\beta$ is a quotient $\alpha / \alpha^{\prime}$ of algebraic numbers conjugate over $K$.

The condition $|O(\sigma, \beta)|=\operatorname{deg} \beta$ also holds for some $\sigma \in G_{\beta}$ when the degree $\operatorname{deg} \beta$ of $\beta$ over $K$ is prime, since in this case $\left|G_{\beta}\right|$ is a multiple of $\operatorname{deg} \beta$, so that, by Cauchy's Theorem, $G_{\beta}$ contains an element of order $\operatorname{deg} \beta$. Hence, for this $\sigma$, $P(\sigma, \beta)=\operatorname{Norm}_{K}(\beta)$, so that $\operatorname{Norm}_{K}(\beta)$ being a root of unity is sufficient to show, by Theorem 1.1, that $\beta$ is a quotient $\alpha / \alpha^{\prime}$ of numbers conjugate over $K$.

The following result follows immediately from Theorem 1.1.

Corollary 1.4. Suppose that $\operatorname{Norm}_{K}(\beta)$ is a root of unity but that no proper subproduct of the conjugates of $\beta$ over $K$ is a root of unity. Then $\beta$ is a quotient $\alpha / \alpha^{\prime}$ of algebraic numbers conjugate over $K$ if and only if $G_{\beta}$ contains a cycle of length $\operatorname{deg} \beta$.

For $\beta$ whose norm over $K$ is a root of unity we would expect that, for most such $\beta$, no subproduct of the conjugates of $\beta$ would be a root of unity. Thus Corollary 1.4 covers generic $\beta$ whose norm is a root of unity. (In the case $K=\mathbb{Q}$, algebraic numbers of norm \pm 1 were called unit-norms in [2]. A unit norm is a unit if and only if it is also an algebraic integer.)

To show that the condition $\operatorname{Norm}_{K}(\beta)$ equal to a root of unity is not sufficient for $\beta$ to be a quotient of conjugates over $K$, consider the following example.

ExAmple 1.5. Let $K=\mathbb{Q}$, and $\beta=1+\sqrt{2}+\sqrt{6}$, with other conjugates $\beta_{2}=1-$ $\sqrt{2}+\sqrt{6}, \quad \beta_{3}=1+\sqrt{2}-\sqrt{6}, \quad \beta_{4}=1-\sqrt{2}-\sqrt{6}$. Then $\beta$ has norm 1 , $N_{\beta}=\mathbb{Q}(\beta)=\mathbb{Q}(\sqrt{2}, \sqrt{3})$, and $G_{\beta}$ is a 4-group. The orbits $O(\sigma, \beta)$ as $\sigma$ takes all four values in $G_{\beta}$ are $\{\beta\},\left\{\beta, \beta_{2}\right\},\left\{\beta, \beta_{3}\right\}$ and $\left\{\beta, \beta_{4}\right\}$, with $P(\sigma, \beta)=\beta, 5+2 \sqrt{6}$, $-3+2 \sqrt{2}$ and $-7+4 \sqrt{3}$ respectively, none of which is a root of unity. Thus, although $\operatorname{Norm}_{\mathbb{Q}}(\beta)=1, \beta$ is not a quotient of conjugates, by Theorem 1.1.

We also remark that $\beta=(2+\sqrt{3})(\sqrt{2}-1)(\sqrt{2}+\sqrt{3})$, where, by Theorem 1.1 , each of the three numbers on the right hand side is a quotient of two conjugates over $\mathbb{Q}$. This shows that the set of numbers which are expressible as quotients of two conjugates over $\mathbb{Q}$ does not form a multiplicative group. 
This next example indicates that an algebraic number $\beta$ can be a quotient of conjugates but not be a quotient of conjugates in $N_{\beta}$.

EXAmple 1.6. Let $K=\mathbb{Q}$, and

$$
\beta=\frac{1}{6}(1+i)(2+\sqrt{2})(1+2 i \sqrt{2})=-\frac{1}{3}+i-\frac{\sqrt{2}}{2}+\frac{5}{6} i \sqrt{2} \in N_{\beta}=\mathbb{Q}(\sqrt{2}, i),
$$

where, for this example, $i=\sqrt{-1}$. We claim that $\beta$ is a quotient of conjugates but is not a quotient of conjugates in $N_{\beta}$. Now $G_{\beta}=\left\{1, \sigma_{2}, \sigma_{3}, \sigma_{2} \sigma_{3}\right\}$, where

$$
\sigma_{2}:\left\{\begin{array}{c}
\sqrt{2} \mapsto-\sqrt{2} \\
i \mapsto i
\end{array}, \quad \sigma_{3}:\left\{\begin{array}{c}
\sqrt{2} \mapsto \sqrt{2} \\
i \mapsto-i
\end{array} .\right.\right.
$$

Now, putting $\beta_{2}=\sigma_{2} \beta$, we have $\beta \beta_{2}=i$ so that, by Theorem 1.1, $\beta$ is a quotient of conjugates. Indeed taking $\beta^{1 / 2}$ and $\beta_{2}^{1 / 2}$, with positive imaginary parts, $\omega_{8}=e^{2 \pi i / 8}$ and $3^{1 / 8}$ the real positive 8 th root of 3 , then $\alpha=3^{1 / 8} \beta^{1 / 2}$ and $\alpha^{\prime}=\omega_{8}^{3} 3^{1 / 8} \beta_{2}^{1 / 2}$ are conjugate, and $\beta=\alpha / \alpha^{\prime}$. (The construction of $\alpha$ and $\alpha^{\prime}$ follows from the method given in Section 5 below, combined with an application of Lemma 3.2. As that proof requires that the prime $p$ (here $p=3$ ) used in the construction should not divide the discriminant $\operatorname{Disc}\left(N_{\beta^{1 / 2}} / \mathbb{Q}\right)$, the smallest $p$ we should take is $p=11$. However, as $3 \nmid \operatorname{Disc}\left(N_{\beta} / \mathbb{Q}\right)$ it turns out to be permissible to take $p=3$.)

The minimal polynomial of $\alpha$ and $\alpha^{\prime}$ over $\mathbb{Q}$ can be calculated, with the help of Maple, to be

$$
z^{32}+\frac{1156}{27} z^{24}+\frac{7572550}{729} z^{16}+\frac{1156}{3} z^{8}+81
$$

(Using $p=11$ produces a polynomial with much larger coefficients.)

On the other hand, suppose that, for some $\alpha \in N_{\beta}, \sigma \in G_{\beta}, \beta=\alpha / \sigma \alpha$. Then $\beta \sigma \beta=1$. However, none of $\beta^{2}, \beta \sigma_{2} \beta, \beta \sigma_{3} \beta$ or $\beta \sigma_{2} \sigma_{3} \beta$ is equal to 1 .

We remark also that if $\beta$ is reciprocal (conjugate to $1 / \beta$ over $\mathbb{Q}$ ) then it is a quotient of conjugates $(1+\beta) /(1+1 / \beta)$. This result, and in fact the same result for $\beta$ antireciprocal (conjugate to $-1 / \beta$ over $\mathbb{Q}$ ) also follows from Theorem 1.1 , because of the existence of an orbit $\{\beta, \pm 1 / \beta\}$.

2. Additive versions of the results. Our second main result, Theorem 2.1 below, is an additive (or perhaps we should say 'subtractive'!) version of Theorem 1.1. As we shall see, the result and its proof are simpler in this case. It is perhaps surprising (but see also [6]) that such multiplicative and additive problems for algebraic numbers can be treated so similarly.

Theorem 2.1. An algebraic number $\beta$ can be written as a difference $\alpha-\alpha^{\prime}$ of algebraic numbers $\alpha, \alpha^{\prime}$ conjugate over $K$ if and only if there is a $\sigma \in G_{\beta}$ such that $S(\sigma, \beta)=0$. If such $\alpha, \alpha^{\prime}$ exist, then they can be chosen to lie in $N_{\beta}$.

Analogous to the Corollaries of Theorem 1.1 we obtain three corollaries to Theorem 2.1.

Corollary 2.2. (Additive Hilbert's Theorem 90) Suppose that $L / K$ is a cyclic Galois extension and that $\beta \in L$. Then $\beta$ is a difference $\alpha-\alpha^{\prime}$ for some $\alpha, \alpha^{\prime} \in L$ conjugate over $K$ if and only if $\operatorname{Trace}_{K}(\beta)=0$. 
This is well known. See, for example [3, Section 2.6]. It is an immediate consequence of the following result.

Corollary 2.3. If $|O(\sigma, \beta)|=\operatorname{deg} \beta$, for some $\sigma \in N_{\beta}$, and $\beta$ has zero trace, then $\beta$ is a difference $\alpha-\alpha^{\prime}$ of numbers $\alpha, \alpha^{\prime}$ in $N_{\beta}$, and conjugate over $K$.

Also, as for Corollary 1.3, we see from Corollary 2.3 that if $\operatorname{deg}(\beta)$ is prime and $\operatorname{Trace}_{K}(\beta)=0$ then $\beta$ is again a difference $\alpha-\alpha^{\prime}$ of conjugates $\alpha, \alpha^{\prime}$ in $N_{\beta}$.

The next corollary covers generic $\beta$ with $\operatorname{Trace}_{K}(\beta)=0$, and is analogous to Corollary 1.4.

Corollary 2.4. Suppose that $\beta$ has Trace $_{K}(\beta)=0$, but that no proper subsum of the conjugates of $\beta$ over $K$ has sum 0 . Then $\beta$ is a difference $\alpha-\alpha^{\prime}$ of algebraic numbers conjugate over $K$ if and only if $G_{\beta}$ contains a cycle of length $\operatorname{deg} \beta$.

We now show that the condition $\operatorname{Trace}_{K}(\beta)=0$ is not sufficient for $\beta$ to be a difference of conjugates.

Example 2.5. Let $K=\mathbb{Q}$ and $\beta=\sqrt{2}+\sqrt{3}+\sqrt{6}$, with $N_{\beta}=\mathbb{Q}(\sqrt{2}, \sqrt{3})$ as in Example 1.5, and $G_{\beta}$ a 4-group. It is easily checked that, although $\operatorname{Trace}_{\mathbb{Q}}(\beta)=0$, $S(\sigma, \beta) \neq 0$ for each $\sigma$ in $G_{\beta}$ so that, by Theorem $2.1, \beta$ is not a difference of conjugates over $\mathbb{Q}$.

Also, note that since each of $\sqrt{2}, \sqrt{3}$ and $\sqrt{6}$ is, by Theorem 2.1, a difference of conjugates over $\mathbb{Q}$, we see that the set of numbers expressible as a difference of conjugates over $\mathbb{Q}$ does not form an additive group over $\mathbb{Q}$.

3. Proof of Theorem 1.1. For the proof we need the following lemmas.

Lemma 3.1. Let $k$ be a positive integer, $F$ be an algebraic number field containing $K$, and $p$ be a prime not dividing the discriminant $\operatorname{Disc}(F / \mathbb{Q})$. Suppose that $\rho, \rho^{\prime} \in F$ are conjugate over $K$. Then they are also conjugate over $K\left(p^{1 / k}\right)$.

Proof. If $p \nmid \operatorname{Disc}(F / \mathbb{Q})$, then $F / \mathbb{Q}$ is unramified at $p$ by [5, p.30]. However, $F\left(p^{1 / k}\right) / F$ then has ramification index $k$ at $p$, so that

$$
\left[F\left(p^{1 / k}\right): F\right]=k
$$

Now suppose that $\rho$ and $\rho^{\prime}$ are conjugate over $K$, with say $[K(\rho): K]=r$. If $\rho$ and $\rho^{\prime}$ are not conjugate over $K\left(p^{1 / k}\right)$, then the minimal polynomial of $\rho$ over $K$ factorizes over $K\left(p^{1 / k}\right)$, with $\rho$ and $\rho^{\prime}$ zeros of different factors, so that $\left[K\left(p^{1 / k}, \rho\right): K\left(p^{1 / k}\right)\right]=r^{\prime}$. Now, on the one hand, as $K(\rho) \subset F$,

$$
\left[K\left(p^{1 / k}, \rho\right): K(\rho)\right]=k
$$

by (1), so that

$$
\left[K\left(p^{1 / k}, \rho\right): K\right]=\left[K\left(p^{1 / k}, \rho\right): K(\rho)\right][K(\rho): K]=k r
$$


while on the other hand

$$
\left[K\left(p^{1 / k}, \rho\right): K\right]=\left[K\left(p^{1 / k}, \rho\right): K\left(p^{1 / k}\right)\right]\left[K\left(p^{1 / k}\right): K\right]=r^{\prime} k<k r,
$$

a contradiction. Hence $\rho$ and $\rho^{\prime}$ are conjugate over $K\left(p^{1 / k}\right)$.

Lemma 3.2. Suppose that, for some positive integer $k, \beta^{k}$ is a quotient $\delta / \delta^{\prime}$ of algebraic numbers $\delta, \delta^{\prime}$ conjugate over $K$. Then $\beta$ is also a quotient $\alpha / \alpha^{\prime}$ of algebraic numbers $\alpha, \alpha^{\prime}$ conjugate over $K$, with $\alpha^{k} \in K(\delta)$.

Proof. Suppose that $\beta^{k}=\delta / \delta^{\prime}$, where $\delta$ and $\delta^{\prime}$ are conjugate over $K$. Fix a $k$ th root $\rho$ of $\delta$, say, with conjugates $\rho=\rho_{1}, \rho_{2}, \ldots, \rho_{s}$ over $K$. Since $\rho_{1}^{k}, \ldots, \rho_{s}^{k}$ include all conjugates of $\rho^{k}=\delta$, some $\rho_{i}$ say $\rho_{2}$, satisfies $\rho_{2}^{k}=\delta^{\prime}$. Then $\beta^{k}=\left(\rho / \rho_{2}\right)^{k}$ implies that $\beta=\varepsilon_{k} \rho / \rho_{2}$ for some $\varepsilon_{k}$, a $k$ th root of unity.

Now let $F$ be the normal closure of $K\left(\rho, \omega_{k}\right)$ over $K$, where $\omega_{k}$ is a primitive $k$ th root of unity. Using (1), choose a prime $p$ such that $F\left(p^{1 / k}\right) / F$ has degree $k$. Note that $F\left(p^{1 / k}\right)$ is Galois over $F$ and over $K$. Hence there is an automorphism $\tau \in$ $\operatorname{Gal}\left(F\left(p^{1 / k}\right) / F\right) \subset \operatorname{Gal}\left(F\left(p^{1 / k}\right) / K\right)$ fixing $F$ and taking $p^{1 / k} \mapsto \varepsilon_{k}^{-1} p^{1 / k}$. Also, by Lemma 3.1, there is an automorphism $\sigma \in \operatorname{Gal}\left(F\left(p^{1 / k}\right) / K\left(p^{1 / k}\right)\right) \subset \operatorname{Gal}\left(F\left(p^{1 / k}\right) / K\right)$ fixing $K\left(p^{1 / k}\right)$ and taking $\rho \mapsto \rho_{2}$. Then

$$
\tau \sigma\left(p^{1 / k} \rho\right)=\tau\left(p^{1 / k} \rho_{2}\right)=\varepsilon_{k}^{-1} p^{1 / k} \rho_{2},
$$

so that $\alpha=p^{1 / k} \rho$ and $\alpha^{\prime}=\varepsilon_{k}^{-1} p^{1 / k} \rho_{2}$ are conjugate over $K$. Hence

$$
\beta=\frac{\varepsilon_{k} \rho}{\rho_{2}}=\frac{p^{1 / k} \rho}{\varepsilon_{k}^{-1} p^{1 / k} \rho_{2}}=\frac{\alpha}{\alpha^{\prime}} .
$$

Finally, note that $\alpha^{k}=p \delta \in K(\delta)$.

We can now prove Theorem 1.1. The construction of $\delta$ below is similar to that of Hilbert in the proof of his Theorem 90 [4]. First suppose that there is a $\sigma \in G_{\beta}$ with $P(\sigma, \beta)$ an $\ell$ th root of unity. Now define the subfield $E$ of $N_{\beta}$ by $E=\left\{x \in N_{\beta}: \sigma x=x\right\}$, the fixed field of $\sigma$. Suppose that $\gamma \in N_{\beta}$ is a primitive element for $N_{\beta} / E$, so that $N_{\beta}=E(\gamma)$. Denote the order of $\sigma$ in $G_{\beta}$ by $n$ and, as $m=|O(\sigma, \beta)|$ divides $n$, write $n=s m$ say. It follows that, on putting $\beta_{i}=\sigma^{i-1} \beta(i=1, \ldots, n)$, we have

$$
\beta_{1} \cdots \beta_{n}=\left(\beta_{1} \cdots \beta_{m}\right)^{s}=P(\sigma, \beta)^{s},
$$

which, for $k=\ell / \operatorname{gcd}(\ell, s)$, is a $k$ th root of unity. Hence $\beta_{1}^{k} \cdots \beta_{n}^{k}=1$.

Now let $h$ be the smallest positive integer such that $\sigma^{h} \gamma=\gamma$. By Galois correspondence, $\left[N_{\beta}: E\right]=n$, so that $\gamma$ has degree $n$ over $E$. Since the coefficients of the polynomial $\prod_{i=1}^{h}\left(X-\sigma^{i-1} \gamma\right)$ are fixed under $\sigma$, the polynomial is in $E[X]$, from which it follows that $h=n$ and the conjugates of $\gamma$ over $E$ are $\gamma_{i}=\sigma^{i-1} \gamma$ $(i=1, \cdots, n)$. Then as the Vandermonde determinant $\operatorname{det}\left(\gamma_{i}^{j}\right)_{i, j=1, \ldots, n}$ is non-zero, it follows that for at least one $j \in\{1,2, \ldots, n\}, \delta=\sum_{i=1}^{n} \gamma_{i}^{j} \prod_{t=1}^{i} \beta_{t}^{k}$ is non-zero. Choosing such a $j$, and using $\beta_{1}^{k} \cdots \beta_{n}^{k}=1$ it is easily checked that $\beta^{k} \sigma \delta=\delta, \beta^{k}=\delta / \sigma \delta$. Then, by Lemma 3.2, $\beta=\alpha / \alpha^{\prime}$ with $\alpha^{k} \in K(\delta) \subset N_{\beta}$. 
Conversely, suppose that $\beta=\alpha / \sigma \alpha$ for some $\sigma \in \operatorname{Gal}\left(N_{\alpha} / K\right)$. Let $m=|O(\sigma, \beta)|$ be the least integer such that $\sigma^{m} \beta=\beta$, and $r$ be the least integer such that $\sigma^{r} \alpha=\alpha$. Then since

$$
\sigma^{r} \beta=\sigma^{r}\left(\frac{\alpha}{\sigma \alpha}\right)=\frac{\sigma^{r} \alpha}{\sigma^{r+1} \alpha}=\frac{\alpha}{\sigma \alpha}=\beta
$$

$m$ divides $r, r=q m$ say. Then on the one hand

$$
\prod_{i=1}^{r} \sigma^{i-1}\left(\frac{\alpha}{\sigma \alpha}\right)=\prod_{i=1}^{r} \sigma^{i-1} \beta=\left(\beta_{1} \ldots \beta_{m}\right)^{q}
$$
while on the other hand this product equals $\prod_{i=1}^{r} \sigma^{i-1} \alpha / \prod_{i=1}^{r} \sigma^{i} \alpha=1$. Hence $\beta_{1} \cdots \beta_{m}$ is a
$q$ th root of unity.

4. Proof of Theorem 2.1. This is an additive version of the proof of Theorem 1.1. It is much simpler, the construction of $\alpha$ being much more straightforward, and no auxiliary lemmas being needed. First suppose that there is a $\sigma \in G_{\beta}$ with $S(\sigma, \beta)=0$. Put $\beta_{i}=\sigma^{i-1} \beta(i=1, \cdots, m)$, where again $m=|O(\sigma, \beta)|$, and

$$
\gamma=(m-1) \beta_{1}+(m-2) \beta_{2}+\ldots+\beta_{m-1},
$$

so that

$$
\sigma \gamma=(m-1) \beta_{2}+(m-2) \beta_{3}+\ldots+\beta_{m}=\gamma-m \beta_{1}
$$

using $\beta_{1}+\ldots+\beta_{m}=0$. Thus $\beta=\alpha-\sigma \alpha$ is a difference of conjugates, where $\alpha=\gamma / m$. Note that $\alpha \in N_{\beta}$.

Conversely suppose that $\beta=\alpha-\sigma \alpha$ for some $\sigma \in \operatorname{Gal}\left(N_{\alpha} / K\right)$. Defining $r$ as in the previous proof, we have

$$
\sigma^{r} \beta=\sigma^{r}(\alpha-\sigma \alpha)=\sigma^{r} \alpha-\sigma^{r+1} \alpha=\alpha-\sigma \alpha=\beta,
$$

so that again $m$ divides $r, r=q m$ say. Then

$$
\sum_{i=1}^{r} \sigma^{i-1}(\alpha-\sigma \alpha)=q\left(\beta_{1}+\ldots+\beta_{m}\right),
$$

but also equals

$$
\sum_{i=1}^{r} \sigma^{i-1} \alpha-\sum_{i=1}^{r} \sigma^{i} \alpha=0
$$

giving $\beta_{1}+\ldots+\beta_{m}=0$.

5. A multiplicative construction for $\alpha$. Our version of Hilbert's construction of $\delta$ in the proof of Theorem 1.1 above, from which $\alpha$ was obtained, used both the 
conjugates of $\beta$ and those of a primitive element $\gamma$. The construction we give now, a multiplicative analogue of that used in the proof of Theorem 2.1 above, is simpler. It is more efficient for the purpose of constructing $\alpha$, as $\gamma$ does not need to be found and used. This was why we applied it in Example 1.6 above. However, Hilbert 90 does not follow as a corollary from this result.

Here is the construction. Suppose that there is a $\sigma \in G_{\beta}$ with $P(\sigma, \beta)$ an $\ell$ th root of unity, $m=|O(\sigma, \beta)|$ and $\beta_{i}=\sigma^{i-1} \beta$ for $i=1, \ldots, m$. Then for $\delta=\beta_{1}^{(m-1) \ell}$ $\beta_{2}^{(m-2) \ell} \cdots \beta_{m-2}^{2 \ell} \beta_{m-1}^{\ell}$ we have $\sigma \delta=\beta_{2}^{(m-1) \ell} \beta_{3}^{(m-2) \ell} \cdots \beta_{m-1}^{2 \ell} \beta_{m}^{\ell}=\beta^{-\ell m} \delta$, using $\beta_{1}^{\ell} \beta_{2}^{\ell} \ldots$ $\beta_{m}^{\ell}=1$, which gives $\beta^{\ell m}=\delta / \sigma \delta$. Hence, applying Lemma 3.2, we again obtain $\alpha$ with $\beta=\alpha / \sigma \alpha$.

Acknowledgements. This work arose from our study of the 'Remak height' ([1],[2]). We thank Bob Odoni for reminding us of the connection with Hilbert's Theorem 90. Also, we gratefully acknowledge the support of the London Mathematical Society, enabling A.D. to visit Edinburgh in Autumn 2000, where this work was done. He was also supported by the Lithuanian State Studies and Science Foundation.

\section{REFERENCES}

1. A. Dubickas, The Remak height for units, Acta Math. Hungar. (submitted).

2. A. Dubickas and C. J. Smyth, On the Remak height, the Mahler measure, and conjugate sets of algebraic numbers lying on two circles, Proc. Edinburgh Math. Soc. (2) 44 (2001), 1-17.

3. K. W. Gruenberg, Profinite groups, in Algebraic number theory, Proc. Instructional Conf., Brighton, 1965 (Academic Press, 1967), 116-127.

4. D. Hilbert, Die Theorie der algebraischen Zahlkörper, Jahresber. Deutsch. Math.Verein. 4 (1897), 175-546. English translation by I.T. Adamson: The theory of algebraic number fields (Springer-Verlag, 1998).

5. G. J. Janusz, Algebraic number fields (Academic Press, 1973).

6. C. J. Smyth, Additive and multiplicative relations connecting conjugate algebraic numbers, J. Number Theory 23 (1986), 243-254. 